\title{
Validation of the Finnish version of the BODY-Q patient-reported outcome instrument among patients who underwent abdominoplasty
}

\author{
J.P. Repo ${ }^{a, *}$, P. Homsy ${ }^{b}$, M.M. Uimonen ${ }^{a}$, R.P. Roine ${ }^{c, d}$, \\ T. Jahkola ${ }^{\text {, P. Popov }}$
}

${ }^{a}$ Department of Surgery, Central Finland Central Hospital, Keskussairaalantie 19, 40620 Jyväskylä, Finland

${ }^{\mathrm{b}}$ Department of Plastic Surgery, University of Helsinki and Helsinki University Hospital, HUS, Finland

' Group Administration, University of Helsinki and Helsinki University Hospital, HUS, Finland

${ }^{\mathrm{d}}$ Department of Health and Social Management, Research Centre for Comparative Effectiveness and

Patient Safety, University of Eastern Finland, Kuopio, Finland

e Eira Hospital, Helsinki, Finland

Received 3 November 2018; accepted 12 February 2019

Available online $x x x$

\section{KEYWORDS}

Abdominoplasty;

BODY-Q;

Body;

Contouring;

Body image;

Quality of life

\begin{abstract}
Summary
Background: Massive weight loss can notably affect patients' health-related quality of life (HRQoL) and body image. Yet, no body contouring specific instruments to assess HRQoL and body image after massive weight loss have been validated in Finnish. The BODY-Q includes 26 independently functioning scales and a single checklist that measure appearance, HRQoL, and experience of care. The aim of the present study was to translate and validate a Finnish version of the BODY-Q among patients who underwent abdominoplasty.

Methods: The BODY-Q was translated into Finnish using recommended guidelines. Eighty-two patients who underwent abdominoplasty due to massive weight loss were identified from hospital records using procedure codes. A postal survey including the BODY-Q, the 15D, and general health and pain instruments was used. Criterion validity, Cronbach's alpha, and floor and ceiling effects were analyzed.

Results: The BODY-Q translated well into Finnish. Fifty-three patients returned the questionnaires (response rate $65 \%$ ) and were included. All but the Scars subscale correlated significantly with the 15D mean score, thus indicating strong criterion validity against a generic HRQoL tool.
\end{abstract}

* Corresponding author.

E-mail address: jussi.repo@ksshp.fi (J.P. Repo).

1748-6815/@ 2019 British Association of Plastic, Reconstructive and Aesthetic Surgeons. Published by Elsevier Ltd. All rights reserved. 
The Excess Skin and the Physical Function scales reached the ceiling effect ( $>15 \%$ of maximum points) in our postoperative sample. No floor effects were observed. Internal consistency of the BODY-Q scales was high (Cronbach's alpha range, 0.81-0.95).

Conclusions: The Finnish version of the BODY-Q instrument is equivalent in terms of content, accuracy, and comprehensiveness to the original English version. The findings of the present study indicate that the BODY-Q has psychometric properties suitable for assessing outcomes and treatment effectiveness of abdominoplasty.

(C) 2019 British Association of Plastic, Reconstructive and Aesthetic Surgeons. Published by Elsevier Ltd. All rights reserved.

\section{Introduction}

Excess skin after weight loss has a notable impact on appearance and health-related quality of life (HRQoL). ${ }^{1}$ Therefore, body contouring surgery for esthetic and healthpromoting purposes after bariatric surgery and massive weight loss can significantly improve patients' body image and HRQoL. ${ }^{2-6}$

In Finland, the single-payer public healthcare currently provides body contouring for patients whose condition of excess skin requires recurring health services. ${ }^{7}$ Indications for operation can be intertrigo, limitations in daily activities, or restrictions in physical activities due to excess skin. ${ }^{7}$ An additional criterion is body mass index (BMI) less than $32 \mathrm{~kg} / \mathrm{m}^{7}$ Body contouring for purely esthetic reasons is not funded by the public healthcare system, but the patient can seek help from the private sector. There are no data available of the amount of body contouring procedures performed annually in Finland or Europe. Although several international studies have shown body contouring surgery for excess skin to have a positive impact on HRQoL and body image, the evidence to support these procedures in Finnish patients is scarce. ${ }^{2,3}$ Furthermore, generic patient-reported outcome (PRO) HRQoL instruments may not be specific enough to capture what is relevant to patients with excess skin.

The BODY-Q was designed to capture what is most relevant for patients undergoing weight loss and/or body contouring. ${ }^{8}$ The original BODY-Q field-test publication included 18 scales and the obesity-specific symptom checklist. ${ }^{8}$ More recently, the BODY-Q๑ has added a Chest Module (chest and nipples scales) field-tested in 689 participants ${ }^{9}$ and a stretch marks scale tested with 630 participants. ${ }^{10}$ The BODY-Q scales and checklist measure appearance $(n=12)$, HRQoL $(n=6)$, and experience of health care $(n=4) .^{8}$

The BODY-Q instrument has extensive potential for assessing different aspects of the impact of body contouring surgery. Nonetheless, to the best of our knowledge, the only foreign language (other than American and British English) validation study of the BODY-Q to date has been that of Poulsen and her team in Danish. ${ }^{11}$ There has been a need for a weight loss and body contouring-specific PRO instrument in Finnish to assess the effectiveness of procedures such as abdominoplasty after massive weight loss to further clarify the importance for these operations in the Finnish public health care system.

The BODY-Q was chosen for linguistic and psychometric validation into Finnish because of its reliability, validity, and responsiveness. ${ }^{1,8,11-13}$ More specifically, the
BODY-Q has undergone a rigorous development process that included 63 patient interviews, 22 cognitive interviews, and input from 9 clinical experts, followed by an international (Canada, USA, and UK) field-test study that included 403 pre- and postweight loss and 331 pre- and postbody contouring surgery patients. ${ }^{8} \mathrm{~A}$ recent systematic review that compared 24 PRO instruments for bariatric and body contouring surgery singled out the BODY-Q as possessing the strongest evidence for quality of measurement properties. ${ }^{12}$ As it can be used to capture what is relevant to the patient, it was recommended to be used in future clinical trials ${ }^{12}$ for assessing the HRQoL and body image related to excess skin after massive weight loss.

The aim of the present study was to investigate the reliability and validity of the Finnish version of the BODY-Q( instrument among patients who had undergone abdominoplasty.

\section{Methods}

The study protocol was approved by the Ethics Committee of Helsinki and Uusimaa Hospital District and the head of the Musculoskeletal and Plastic Surgery Research Center, Helsinki, Finland. Patients who had undergone abdominoplasty from 2009 to 2017 were identified from hospital records using the Nordic Medico-Statistical Committee (NOMESCO) procedure code QBJ30 Correction of Abdominal Apron. Inclusion criteria were complete understanding of written Finnish, age at least 18 years, and previous abdominoplasty procedure due to massive weight loss. Patients who underwent abdominoplasty postpregnancy or due to hernia were excluded. Altogether, 82 matching massive weight loss patients were identified from hospital records.

Patients were approached through mail by sending a questionnaire package that included the BODY-Q, the 15D, visual analog scale to measure pain and general health, and clinical and demographic questions. Patient records were reviewed for the indication of the body contouring procedure, the amount of weight loss before the operation, whether the patient had undergone bariatric surgery before the body contouring, date of the body contouring procedure, and age and sex of the participants. Participants signed an informed consent according to the Declaration of Helsinki and returned the completed questionnaires together with the signed consent in a prepaid envelope. No further contact was made. 


\section{Instruments}

\section{BODY-Q๑}

The original BODY-Q set of 18 scales and obesity-specific symptom checklist, which measure appearance $(n=9)$, HRQoL $(n=5)$, and patient experience of care $(n=4)$, was used. The Appearance scales assess satisfaction with different parts of the body (Abdomen, Arms, Back, Body, Buttocks, Inner thighs, or Hips and Outer Thighs) as well as how bothered someone is with Excess Skin and body contouring Scars. The HRQoL scales measure Body image, Physical functioning, Physical symptoms, Psychological functioning, Sexual functioning, and Social functioning. Patient experience measures Doctor/Surgeon, Information, Medical team, and Office staff.

The BODY-Q scales function independently, and it is recommended that only relevant scales are used depending on the patient population and/or research question being asked. Because the focus of our study was on abdominoplasty, participants were asked to complete the Abdomen, Scars, and Excess skin appearance scales alongside all HRQoL scales, which best serve for abdominoplasty patients. The BODY-Q scores can be converted to a scale from 0 to 100 .

\section{D instrument}

The $15 D$ is a valid and reliable, generic self-administered HRQoL instrument containing the following 15 dimensions: Moving, Seeing, Hearing, Breathing, Sleeping, Eating, Speech, Excretion, Usual activities, Mental function, Discomfort and symptoms, Depression, Distress, Vitality, and Sexual activity. ${ }^{14}$ Patients choose one of the five levels in each dimension that best describes their current state of health (1-5; best to worst possible score). This study used the 15D single index score, which represents the overall HRQoL. The score ranges from 0 to 1 , with 0 equivalent to being dead and 1 being the best imaginable HRQoL state. The 15D was chosen for criterion validity testing of the Finnish version of the BODY-Q, as the 15D instrument is a widely adopted instrument in Finland, ${ }^{15}$ and it has previously been used to assess the treatment effectiveness of excess skin using abdominoplasty procedure in Finnish patients. ${ }^{2,3}$

\section{Sociodemographic and clinical data questionnaire}

Information of participants' height, weight, occupational details, smoking, and alcohol consumption habits was obtained. A visual analog scale ranging from 0 to $100 \mathrm{~mm}(0-$ $100 \mathrm{~mm}$; best to worst) was used to measure participants' self-reported general health and pain intensity during the preceding week.

\section{Translation and cross-cultural adaptation}

The linguistic validation of the 18 BODY-Q scales and checklist adhered to the International Society for Pharmacoeconomics and Outcomes Research (ISPOR) guidelines. ${ }^{16}$ The ISPOR translation process is described in more detail elsewhere. ${ }^{16}$ Two separate certified, professional native Finnish translators produced two forward-translations leading to a reconciliation version of these two versions. A professional native English translator fluent in Finnish and familiar with the Finnish culture produced a back-translation of the
Finnish version 1. A back-translation panel and a steering group of three surgeons assessed the whole process on separate occasions. Five patients participated in pilot-testing the proposed Finnish version and were later interviewed to reveal any discrepancies in the questionnaire. Pretesting of the instrument with five patients was done by adhering to the ISPOR guidelines. ${ }^{16}$ These five patients completed each of the scales. The committee assessed the pretesting and cognitive debriefing outcomes. A linguistic expert proofread the final version.

\section{Statistical analysis}

Clinical, sosiodemographic, and PRO instrument data are presented as mean with standard deviation (SD), 95\% confidence intervals $(95 \% \mathrm{Cl})$, or as numbers with percentages. The BODY-Q scale scores were converted into 0-100 scores, with higher scores indicating a better outcome. Normality of the data for BODY-Q scales was assessed. Missing values for single variables were imputed adhering to the mean value of other items in the subscale.

Scale targeting was investigated calculating the floor and ceiling values (precentage of minimun and maximum points). A floor or ceiling effect was considered to be confirmed if $15 \%$ of patients scored minimum or maximum points. ${ }^{17}$ Cronbach's alpha served for assessing internal consistency for the BODY-Q subscales.

Linearity with the $15 \mathrm{D}$ was assessed to reveal the relationships between the most relevant subscales for patients who underwent abdominoplasty. The authors hypothesized that the generic HRQoL would have a robust linear relationship with BODY-Q subscales of interest.

Spearman correlation coeffient was used to identify correlation between the $15 \mathrm{D}$ mean score and the subscales of the BODY-Q. Bootstrap method with 5000 replications was used to obtain $95 \% \mathrm{Cls}$. The authors hypothesized that there would be a $>0.50$ correlation between the Abdomen scale and the 15D scores. The information of the strongest correlation with each of the BODY-Q scales was used to choose the appropriate reference measure for criterion validity assessment with the Spearman correlation.

Furthermore, linear regression analyses were used to identify the appropriate predictors of the BODY-Q standardized by age, postoperative $\mathrm{BMI}$, and gender. The regression coefficient $\beta$-value showed how strongly each predictor variable influenceed the criterion variable. The authors measured the $\beta$-value in units of SD. Cohen reference values are $0.1,0.3$, and 0.5 for small, moderate, and strong correlations, respectively.

Analyses were performed using SPSS 25.0 and R. Results are reported adhering to the COSMIN guidelines. ${ }^{18}$

\section{Results}

\section{Translation and adaptation}

The BODY-Q translated well into Finnish. The backtranslation revealed minor linguistic differences in some adjectives compared to the original English version. The differences were addressed and discussed with the questionnaire developer. A translation review panel and a steering group of three surgeons who were fluent in 
Table 1 Patients' sociodemographic and clinical details.

\begin{tabular}{ll}
\hline & $N=53$ \\
\hline Age, years (SD) & $48.8(12.4)$ \\
Women, $n$ (\%) & $45(84.9)$ \\
BMI before operation, mean (SD) & $31.2(6.2)$ \\
Indication for operation, $n$ (\%) & \\
$\quad$ Excess skin, esthetic & $47(88.7)$ \\
$\quad$ Weight loss & $32(60.4)$ \\
$\quad$ Postbariatric & $15(28.3)$ \\
Hygienic problems or eczema due & $6(11.3)$ \\
$\quad$ to excess skin & \\
15D score, mean (SD) & $0.88(0.09)$ \\
BODY-Q and other scales, mean (SD) & \\
Arms & $48.1(27.9)$ \\
Back & $52.4(27.8)$ \\
Body & $46.6(16.4)$ \\
Buttocks & $62.3(25.6)$ \\
Inner thighs & $51.1(36.6)$ \\
Hips and outer thighs & $49.0(24.2)$ \\
\hline
\end{tabular}

Finnish and English each reviewed the different versions of the translations and minor adjustments were made. The pilot-testing and cognitive debriefing interviews with five patients indicated good external validity. Specifically, patients described the Finnish version of the BODY-Q as understandable and easy to complete. The pilot-testing and interviews did not lead to changes. No further adjustments were required, and the final version was completed with due proofreading by a linguistic expert.

\section{Patients}

Fifty-three patients returned the questionnaires and were included in the analysis. Response rate was 65\%. Mean (SD) weight loss before abdominoplasty was 48.3 (15.9) kg. Altogether, 45 (85\%) patients were women. Indication for operation was excess skin due to massive weight loss $(n=47)$ or hygienic problems or eczhema due to excess skin after weight loss $(n=6)$. Patients' sociodemographic and clinical data and other BODY-Q Appearance scale scores unrelated to abdominoplasty are presented in Table 1.

\section{Internal consistency}

Cronbach's alpha ( $\mathrm{Cl}$ 95\%) exceeded 0.90 in all but one subscale as in physical symptoms subscale, the alpha was 0.87 (0.82-0.91). The Cronbach alpha values indicated excellent internal consistency in all but Physical symptoms subscale, in which the consistency was still good (Table 2).

\section{Criterion validity}

Results of Spearman correlation matrix of BODY-Q subscales with bootstrapped $95 \% \mathrm{Cls}$ and the $15 \mathrm{D}$ mean score and Mental health score are presented in Table 3. All BODY-Q subscales, with the exception of the Scars scale, correlated significantly with the 15D score (Figure 2). Spearman correlation coefficients of 15D and Abdomen, Body image, Physical function, and Psychological subscales exceeded 0.5, indicating moderate to strong correlation. Additionally, correlations between the 15D mean score and Abdomen, Excess skin, and Social subscales were weak to moderate. The Scars subscale did not correlate with the 15D score. Only the Social subscale correlated significantly with the 15D dimension of Mental health.

The 15D mean score had a very strong relationship with BODY-Q scale of physical symptoms as revealed by the $\beta$ value. All relationships of the BODY-Q subscales and the 15D are presented in Figure 3.

\section{Normality of the data}

In the subscales of Scars and Physical function, the distribution was skewed to the right with higher scores. The distribution of scores in the Appearance of abdomen and Appearance of excess skin subscales was bimodal (Figure 1).

\section{Floor and ceiling effect}

In the Scars, Physical function, Psychological, and Social subscales, none of the patients received the lowest score, and in the Abdomen, Excess skin, and Body image subscales, the percentage of minimum values was low: $3.8,7.5$, and 7.5 , respectively (Table 2 ). Some of the patients scored the maximum in every subscale. Ceiling values were found in Physical function and Scars subscales with percentages of 32.7 and 36.5 , respectively (Table 2 ).

Table 2 Mean BODY-Q scores of postoperative patients, Cronbach's alphas, and percentage of maximum and minimum points.

\begin{tabular}{lllll}
\hline Category & Mean $(\mathrm{SD})$ & Alpha $(95 \% \mathrm{Cl})$ & Maximum $(\%)$ & Minimum $(\%)$ \\
\hline Abdomen & $51.6(25.1)$ & $0.95(0.93-0.97)$ & 3.8 & 3.8 \\
Scars & $78.4(24.3)$ & $0.95(0.92-0.97)$ & 36.5 & 7.5 \\
Excess skin & $51.5(31.2)$ & $0.95(0.91-0.97)$ & 0.0 & 7.5 \\
Body image & $36.9(25.1)$ & $0.94(0.91-0.96)$ & 32.7 & 7.5 \\
Physical function & $80.6(22.8)$ & $0.91(0.85-0.94)$ & 5.8 & 0.0 \\
Psychological & $66.2(24.2)$ & $0.95(0.92-0.97)$ & 5.9 & 0.0 \\
Sexual & $48.9(25.7)$ & $0.81(0.68-0.89)$ & 9.4 & 5.9 \\
Social & $67.9(22.2)$ & $0.94(0.90-0.96)$ & 1.9 & 0.0 \\
Physical symptoms & $66.3(19.6)$ & $0.86(0.79-0.90)$ & 0.0 \\
\hline
\end{tabular}


Table 3 Spearman correlations with bootstrapped 95\% Cls of BODY-Q subscale scores, 15D score, and mental health dimension score.

\begin{tabular}{lll}
\hline $\mathrm{r}(95 \% \mathrm{Cl})$ & 15D score & 15D Mental health \\
\hline Abdomen & $0.549(0.299-0.754)^{* *}$ & $-0.187(-0.419-0.072)$ \\
Scars & $0.342(0.064-0.571)^{*}$ & $-0.231(-0.490-0.066)$ \\
Excess skin & $0.452(0.212-0.666)^{* *}$ & $-0.350(-0.565 \text { to }-0.134)^{*}$ \\
Body image & $0.663(0.438-0.821)^{* *}$ & $-0.336(-0.528 \text { to }-0.115)^{*}$ \\
Physical function & $0.654(0.435-0.803)^{* *}$ & $-0.051(-0.305-0.186)$ \\
Psychological & $0.662(0.420-0.814)^{* *}$ & $-0.237(-0.461$ to -0.029$)$ \\
Sexual & $0.319(0.040-0.564)^{*}$ & $-0.219(-0.477-0.058)$ \\
Social & $0.530(0.242-0.735)^{* *}$ & $-0.316(-0.524 \text { to }-0.069)^{*}$ \\
Physical symptoms & $0.495(0.217-0.704)^{* *}$ & $0.072(-0.214-0.349)$ \\
\hline
\end{tabular}

$$
\begin{aligned}
& { }^{*} p \text {-value }<0.05 . \\
& { }^{* *} p \text {-value }<0.01 .
\end{aligned}
$$
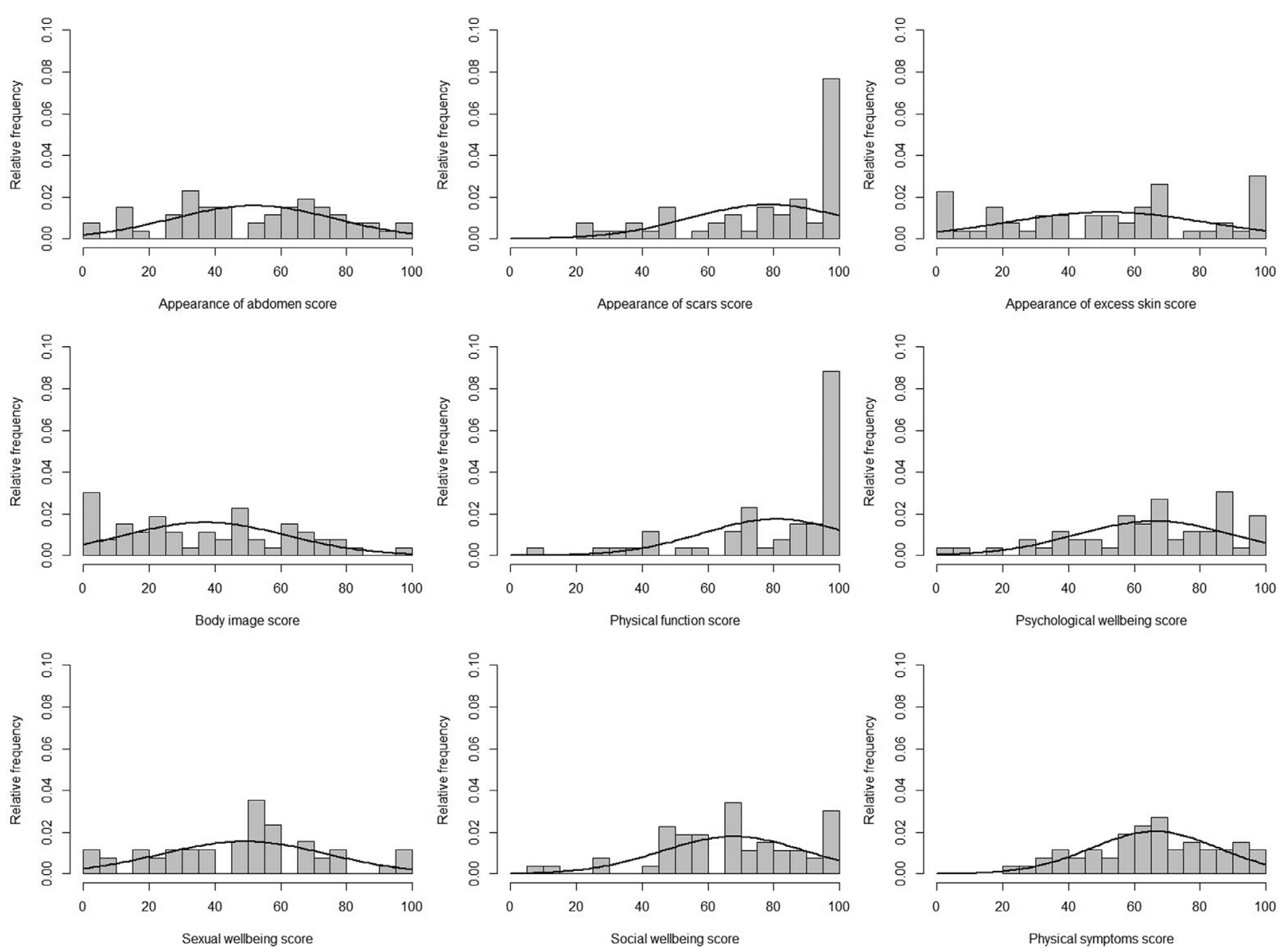

Figure 1 Distribution of scores for the selected BODY-Q subscales. The line illustrates a normal distribution. On the $Y$-axis, the values refer to relative frequency, and on the $X$-axis, the scores of the subscale are presented.

\section{Discussion}

The BODY-Q translated well into Finnish. The present study suggests that the Finnish version of the BODY-Q can provide valid scores for assessing outcomes after abdominoplasty due to massive weight loss. Some ceiling effect was noted in the subscales of Physical function and Scars, indicating potential problems in the content validity in assessing the long-term outcomes in this population (Figure 1).
There was a need for a specific instrument for massive weight loss and body contouring surgery in the Finnish language. The BODY-Q was chosen for translation and quantitative psychometric testing, as it has proven to be reliable, valid, and have responsive qualities, which are considered the most essential ones for a PRO instrument to be psychometrically sound.

The present linguistic validation study of the BODY-Q instrument showed no disease-specific, health-related, or 

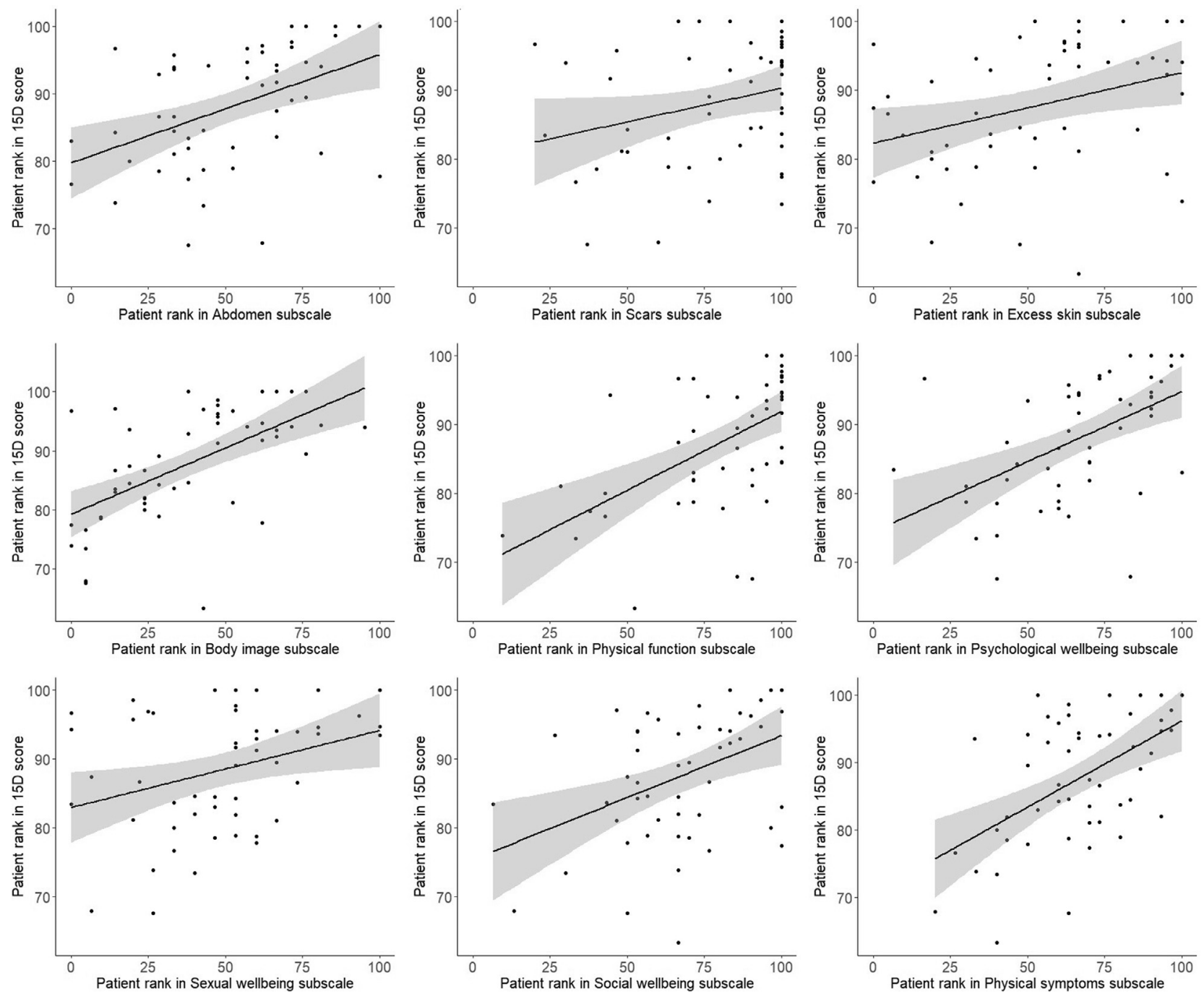

Figure 2 Linearity between the 15D score and each of the BODY-Q subscales. The gray area shows $95 \% \mathrm{Cls}$.

cultural differences between the original and the translated Finnish version of the BODY-Q. Mainly minor linguistic and grammatical changes were needed in the process of translation. Every new instrument should undergo a translatability assessment to reveal any problems in the item content when applied to other languages. ${ }^{19}$ Based on the findings presented here, the BODY-Q had good translatability into a non-Anglo-Saxon language. Thus, the Finnish version of the BODY-Q instrument can be considered equivalent in terms of content, accuracy, and comprehensiveness to the original English version of the BODY-Q.

The BODY-Q provides a set of scales focusing on topics that are relevant to massive weight loss and body contouring patients. Specific issues in massive weight loss and body contouring surgery may not be covered in generic HRQoL instruments, which tend to overlook appearance and body image issues. As the present study used only the 15D instrument, the results are not directly comparable to results of other generic HRQoL instruments, such as the Short Form-36 and the EQ-5D, which are often used in bariatric research. ${ }^{20}$

The BODY-Q scales provided good coverage and targeting in this study for patients who underwent abdominoplasty.
The distribution of scores was good in seven of the nine subscales, which were chosen for their relevance in abdominoplasty. Two subscales (Excess skin and Physical function) exceeded the ceiling effect threshold. This is probably due to the nature of the study design, as the patients were studied postoperatively after having lost considerable weight. They also underwent abdominoplasty to remove excess skin and were not that bothered by excess skin after the procedure. Specifically, there would likely not have been this many maximum points in the Excess Skin scale if the patients had completed the questionnaire before the operation as the findings of Poulsen et al. ${ }^{21}$ suggest. The function of these patients might have also been lower before the operation, as one of the indications in Finland for abdominoplasty is limitation in daily activities and physical activities. On the basis of the findings, these patients had relatively good physical function at the time of completing the questionnaires.

In the present study, Cronbach's alpha ranged between 0.87 and 0.96 . The slightly lower Cronbach's alpha values are probably due to the specific patient selection. Nonetheless, the outcomes can be considered to be in line with those reported in previous studies, thus indicating high internal 


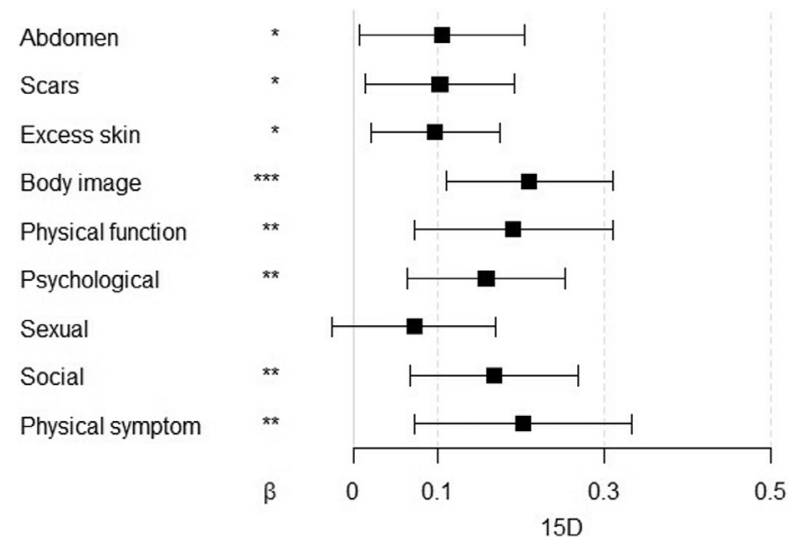

Figure 3 Predictors of the 15D for age-adjusted, BMIadjusted, and gender-adjusted regression coefficients $\beta$. Values of $( \pm) 0.10,0.30$, and 0.50 represent small, moderate, and large relationships, respectively. The boxplot indicates mean $\beta$, and the whiskers indicate $95 \%$ confidence intervals.

consistency. The English versions of the BODY-Q subscales had a Cronbach's alpha (internal consistency) ranging from 0.90 to 0.98 among a heterogeneous population of weight loss patients and body contouring patients. Poulsen et al. also found similar internal consistency in their psychometric study of the BODY-Q. ${ }^{9}$ On the basis of these results, it would seem that the items in each of the scales are closely related.

The present psychometric analysis further investigated the criterion validity of the BODY-Q among a specific abdominoplasty sample. The results showed that the generic HRQoL had statistically significant correlation with most of the BODY-Q subscales investigated in the present study. Linearity illustrations were in line with these results. On the basis of these results, the BODY-Q subscales seem to reflect well patients' HRQoL. The BODY-Q has previously been validated among a representative population of massive weight loss and body contouring patients. This study further vaidated the instrument for assessing outcomes in abdominoplasty patients.

The Finnish version of the BODY-Q can be used for health care quality controls and charting patients' current or changing HRQoL and body image status. Future prospects will include longitudinal follow-up data collection from patients undergoing body contouring surgery. In addition to the data presented here, the BODY-Q Chest Module ${ }^{9}$ has recently been introduced, and it has been translated and adapted into Finnish. ${ }^{22}$ Validation of the Finnish version of the BODY-Q instrument allows participation in the BODY-Q Network, with an aim to evaluate the effectiveness of body contouring surgery as a multinational, multicenter joint venture.

To the best of our knowledge, the present study is the first to evaluate the criterion validity of the BODY-Q instrument. An additional strength of our study is that the authors validated the BODY-Q for a specific group of patients providing valuable information about the measurement properties of the BODY-Q among patients who underwent abdominoplasty. This information can be further used when analyzing future data obtained from patients after abdominoplasty.
The somewhat small sample size can still be considered sufficient for psychometric analyses. ${ }^{17}$ A larger sample size would have, however, allowed to use the Rasch analysis or item response theory to further investigate the psychometric properties of the BODY-Q among this particular patient sample. Furthermore, the present study did not assess the test-retest reliability or responsiveness of the Finnish version of the BODY-Q instrument. The use of the SF-36 or the IWQoL Lite insruments could have provided further insight of the criterion validity of the BODY-Q among abdominoplasty patients.

\section{Conclusions}

The translation of the Finnish version of the BODY-Q followed robust translation and cross-cultural adaptation guidelines. Translatability of the BODY-Q into Finnish was good, as only minor adjustments were needed during the translation process. The Finnish version of the BODY-Q proved to have good internal consistency and strong criterion validity with generic HRQoL. Some ceiling effect was noted in the subscales of Physical funtion and Scars. The comprehensive BODY-Q seems to be a useful tool for assessing appearance, HRQoL, and patient experience after weight loss in patients who underwent abdominoplasty.

\section{Acknowledgments}

The authors thank Heli Sarpila, Leena Caravitis, and Outi Malkavaara for participation in the data collection. The authors are grateful to Paula H. Bergman for statistical consulting.

\section{Funding}

The present study was funded by the Musculoskeletal and Plastic Surgery Research Center Helsinki, Helsinki University Hospital, and University of Helsinki, Finland.

\section{Declaration of interests}

The authors report no conflicts of interest.

\section{References}

1. Klassen AF, Kaur M, Breitkopf $T$, et al. Using the BODY-Q to understand impact of weight loss, excess skin, and the need for body contouring following bariatric surgery. Plast Reconst Surg 2018;142:77-86.

2. Saariniemi KM, Salmi AM, Peltoniemi HH, et al. Abdominoplasty improves quality of life, psychological distress, and eating disorder symptoms: a prospective study. Plast Surg Int 2014; 2014:197232.

3. Saariniemi KM, Salmi AM, Peltoniemi HH, et al. Does liposuction improve body image and symptoms of eating disorders. Plast Reconst Surg Global Open 2015;3(7):e461.

4. de Brito MJA, Nahas FX, Barbosa MVJ, et al. Abdominoplasty and its effect on body image, self-esteem, and mental health. Annal Plast Surg 2010;65:5-10. 
5. van der Beek ES, Geenen R, de Heer FA, et al. Quality of life long-term after body contouring surgery following bariatric surgery: sustained improvement after 7 years. Plast Reconst Surg 2012;130:1133-9.

6. de Zwaan M, Georgiadou E, Stroh CE, et al. Body image and quality of life in patients with and without body contouring surgery following bariatric surgery: a comparison of pre-and post-surgery groups. Front Psychol 2014;5:1310.

7. Setälä LKO, Kaarela O. Postbariatric surgery in the Nordic countries. Finn J Med 2013;23:1728-33.

8. Klassen AF, Cano SJ, Alderman A, et al. The BODY-Q: a patient-reported outcome instrument for weight loss and body contouring treatments. Plast Reconst Surg Global Open 2016;4.

9. Klassen AF, Kaur M, Poulsen L, et al. Development of the BODY$Q$ chest module evaluating outcomes following chest contouring surgery. Plast Reconstr Surg 2018 2018/09/12. doi:10.1097/prs. 0000000000004978.

10. Poulsen L, Pusic A, Robson S, et al. The BODY-Q stretch marks scale: a development and validation study. Aesth Surg J 2018;1:8.

11. Poulsen $L$, Klassen $A$, Rose $M$, et al. Psychometric validation of the BODY-Q in Danish patients undergoing weight loss and body contouring surgery. Plast Reconst Surg Global Open 2017;5(10):e1529.

12. de Vries C, Kalff M, Prinsen C, et al. Recommendations on the most suitable quality-of-life measurement instruments for bariatric and body contouring surgery: a systematic review. Obes Rev 2018;19(10):1395-411.

13. Klassen AF, Cano SJ, Kaur M, et al. Further psychometric validation of the BODY-Q: ability to detect change following bariatric surgery weight gain and loss. Health $Q$ Life Outcom 2017; 15:227.

14. Sintonen $\mathrm{H}$. The 15D instrument of health-related quality of life: properties and applications. Annal Med 2001;33:328-36.
15. Sintonen H. An approach to measuring and valuing health states. Soc Sci Med Part C Med Econ 1981;15:55-65.

16. Wild D, Grove A, Martin M, et al. Principles of good practice for the translation and cultural adaptation process for patient-reported outcomes (PRO) measures: report of the ISPOR Task force for translation and cultural adaptation. Value Health 2005; 8:94-104.

17. McHorney CA, Tarlov AR. Individual-patient monitoring in clinical practice: are available health status surveys adequate. $Q$ Life Resh 1995;4(4):293-307.

18. Prinsen CAC, Mokkink LB, Bouter LM, et al. COSMIN guideline for systematic reviews of patient-reported outcome measures. Qual Life Res 2018;27(5):1147-57.

19. Acquadro C, Patrick DL, Eremenco $S$, et al. International SOCIETY FOR QUALITY OF LIFE RESEARCh (ISOQOL) Translation and Cultural Adaptation Special Interest Group (TCA-SIG). Emerging good practices for Translatability Assessment (TA) of PatientReported Outcome (PRO) measures. J Patient Rep Outcom 2017;2(1):8. doi:10.1186/s41687-018-0035-8.

20. Tayyem R, Ali A, Atkinson J, et al. Analysis of health-related quality-of-life instruments measuring the impact of bariatric surgery. Patient Patient Cent Outcom Res 2011;4:73-87.

21. Poulsen L, Klassen A, Rose $M$, et al. Patient-reported outcomes in weight loss and body contouring surgery: a crosssectional analysis using the BODY-Q. Plast Reconst Surg 2017; 140:491-500.

22. Repo JP, Klassen A, Homsy P, Lindford A, Popov P. Translation and cultural adaptation of the Body-Q Chest module into finnish for use in gynecomastia, weight loss and FTM chest surgery. Value Health 2017;9(20):A766. https://doi.org/10.1016/j.jval. 2017.08.2182. 